\title{
Toward green sensor field by optimizing power efficiency using D-policy M/G/1 queuing systems
}

\author{
Fuu-Cheng Jiang ${ }^{\mathrm{a}}$, Hsiang-Wei $\mathrm{Wu}^{\mathrm{b}}$, Fang-Yi Leu ${ }^{\mathrm{a}}$ and Chao-Tung Yang ${ }^{\mathrm{a}, *}$ \\ ${ }^{a}$ Department of Computer Science, Tunghai University, Taichung Boulevard, Taichung, Taiwan \\ ${ }^{\mathrm{b}}$ Department of Computer Science and Engineering, National Chung-Hsing University, Taichung, \\ Taiwan
}

\begin{abstract}
Power efficiency is a crucially important issue in the IEEE 802.15.4/ZigBee sensor networks (ZSNs) for majority of sensor nodes equipped with non-rechargeable batteries. To increase the lifetime of sensor networks, each node must optimize power consumption as possible. Among open literatures, much research works have focused on how to optimally increase the probability of sleeping states using multifarious wake-up strategies. Making things different, in this article, we propose a novel optimization framework for alleviating power consumption of sensor node with the D-policy M/G/1 queuing approach. Toward green sensor field, the proposed power-saving technique can be applied to prolong the lifetime of ZSN economically and effectively. For the proposed data aggregation model, mathematical framework on performance measures has been formulated. Data simulation using MATLAB tool has been conducted for exploring the feasibility of the proposed approach. And also we analyze the average traffic load per node for tree-based ZSN. Focusing on ZigBee routers deployed at the innermost shell of ZSN, network simulation results validate that the proposed approach indeed provides a feasibly cost-effective approach for prolonging lifetime of ZSNs.
\end{abstract}

Keywords: Queuing theory, optimization, ZigBee sensor network, IEEE 802.15.4 standard

\section{Introduction}

Wireless sensor network (WSN) has emerged as a promising research area for an ever-increasing need for various applications, such as habit or environmental monitoring, wildlife tracking, disaster managing, patient healthcare, humanitarian logistics and industrial data acquisition, and so on [1-4]. A typical WSN is comprised of a large number of distributed sensor nodes with an information collector, referred to as the sink node. These sensor nodes may consist of nothing more than a sensing unit, a battery and a radio server. In this article, from the viewpoint of energy-saving approach in green IT [5], the term "green sensor field (GSN)" is coined to manifest the better energy efficiency than most of plain sensor fields [6]. Hence, one of major design issues toward GSN is to manage power consumption efficiently and to increase the operational lifetime of GSN as possible.

The significant development of WSN has been enforced by the release of IEEE 802.15.4/ZigBee Sensor Network (ZSN) standards. The IEEE 802.15.4 standard [7] defines the medium access control

\footnotetext{
${ }^{*}$ Corresponding author: Chao-Tung Yang, Department of Computer Science, Tunghai University, No. 1727, Section 4, Taichung Boulevard, Taichung 40704, Taiwan. E-mail: ctyang@ thu.edu.tw.
} 
(MAC) layer and the physical (PHY) layer for low-rate, wireless personal area network (LR-WPAN). IEEE 802.15.4 addresses the protocol and compatible interconnection for data communication devices which are usually small, inexpensive, and of limited battery power. Based on the installed capabilities of communication devices, they can be categorized into full function devices (FFDs) and reduced function devices (RFDs). FFDs can operate in three modes serving as a personal area network (PAN) coordinator, a coordinator, or a device. An FFD can talk to RFDs or other FFDs, while an RFD can talk only to an FFD. FFDs are capable to forward frames for other devices, while RFDs are without such capability. An RFD is intended for applications that are simpler, such as a temperature/pressure sensor, a light switch, or a passive infrared sensor.

IEEE 802.15.4 standard defines only two bottom layers (PHY and MAC layers) from the viewpoint of protocol stack in the communication network whereas upper layers are defined by ZigBee Alliance. The ZigBee standard [8] is developed for the family of LR-WPANs, allowing network creation, topology formation, network management, and data packet communication over a wireless medium. The combination of IEEE 802.15.4 and ZigBee specifications allows defining a WSN protocol stack capable of supporting a variety of WSN application [9], where such a WSN is also termed as ZigBee sensor network (ZSN). The ZigBee standard extends the generic star topology of an IEEE 802.15.4 LR-WPAN to a tree or mesh structure. In a tree topology, the root (called ZigBee Coordinator: ZC) and all internal nodes are FFDs (called ZigBee Router: ZR), while RFDs can only be leaf nodes named as ZigBee End Devices (ZEDs).

Topologically, the tree structure has been adopted for data collection in WSNs, while all sensor nodes forwarding their traffic load following a data packet gathering tree [10] to the sink node. If IEEE 802.15.4/ZigBee is employed to implement a WSN, the sink node is logically the ZC while other sensor nodes are ZRs and ZEDs. The WSN can be established in the form of a ZigBee tree with ZC as the sensor sink, the ZRs as internal nodes and ZEDs as leaves. The ZR-type sensor node usually behaves as both packet originator and packet router. All of the data generated must eventually reach a single ZC (i.e., sink node) in sensor network. The traffic follows a many-to-one, clustering pattern, where nodes nearer to the ZC carry heavier traffic loads. Therefore, the nodes around the ZC would deplete their energy faster, leading to what is known as an energy hole problem (EHP) around the sink [11].

No more data packets can be delivered to the ZC in case an energy hole appears. Consequently, a considerable amount of energy is wasted, and the network lifetime ends prematurely. For large WSN in the single static sink model, the simulated experiments [12] show that up to $90 \%$ of total initial energy can be left unused when the network lifetime is over. Also with analytical results, Wadaa et al. [13] argue that by the time the sensors one hop away from the sink exhaust their energy budget, sensors farther away (e.g. in the seventh shell) still have up to 93\% of their initial energy budget. The EHP is an inherent risk in ZSN, and emerges to be a constant threat to lifetime security in ZSN.

To alleviate the unavoidable EHP embedded in a tree-based ZSN, we provide an effective and feasible power-saving technique using D-policy M/G/1 queuing theory [14]. A queue threshold, D, is specified for the concept of "queue-based triggering". This threshold could be used to control the total average times of turning on the transmitting function of radio server for the buffered data packets. In the "queuedbased triggering" scheme, when the queue holds D payloads, the sensor node triggers its transmitting function of radio server, and starts the transmission process for the queued packets in a burst.

Three key contribution of this article is as follows: (i) with little management cost, we provide the ZSN administrator with a feasible and economical power-saving technique to prolong operational lifetime of ZSN by alleviating EHP. The D-policy M/G/1 queuing model is expressed in terms of a Petri Net design for qualitative analysis. Relevant mathematical expressions are derived for the power consumption of the 
generic ZigBee device. Data simulations with MATLAB tool imply the feasibility of optimizing energy efficiency. (ii) We analyze the average traffic load on ZRs and ZEDs at each depth in terms of neat closed-form mathematical expressions. (iii) Comprehensive network simulations with NS2 simulator are conducted on verifying the existence of optimizing power consumption by optimizing data aggregation. The simulated results indicate that the proposed approach may provide a feasibly cost-effective approach to elongate the operational lifetime for the ZSN.

The rest of the paper is organized as follows. Section 2 describes related work. In Section 3, a Petri Net design has been proposed firstly for qualitative analysis. Then the D-policy M/G/1 queuing model is adopted and elaborated, and we derive the relevant system performance measures. The optimal D policy is further addressed in terms of total average power consumption, which data simulations are conducted as well for the feasibility of the proposed scheme. Following this, in Section 4, we conduct the mathematical analysis of average traffic load on ZRs and ZDs. In Section 5, focusing on the treebased ZSN, typical design scenario has been illustrated, and the average traffic load per node is simulated for comparison with theoretical values. The network experiments with NS2 simulator are also conducted to verify the proposed technique. Finally, some concluding remarks are made in Section 6.

\section{Related work}

A number of research issues for mitigating the power consumption of WSN/ZSN have been explored in recent years. To minimize the energy consumption of the communication unit, major power-saving techniques can basically be categorized in two groups: power saving through duty cycling and innetwork processing [15]. In-network processing takes advantage of compression or aggregation technique to reduce the number of information to be sent. Duty cycling schemes denotes coordinated sleep/wakeup schedules among nodes in WSN/ZSN. Wakeup strategy determines the time point when to wake up a node from an idle state to the busy state. Basically, scheduling sensor node into sleep mode (shutting down radio server) would inevitably incur not only the management cost including coordination cost among nodes but also the packet-loss risk from shutting the transceiver.

Most MAC protocols for wireless sensor networks have been based on conventional wireless protocols, especially the IEEE 802.11 standard [16]. The IEEE 802.11 standard specifies a CSMA/CA (Carrier Sense and Multiple Access/Collision Avoidance) protocol for reducing the collision probability in multiple accesses. The adopted exponential backoff mechanism can effectively provide the basic function for collision avoidance. This backoff can only reduce the collision probability among nodes competing for the access right of air channel. However it does not address the issue how to alleviate the total average times of medium contention for each sensor node. In other words, it starts packet transmission as long as there is any one arriving packet in its buffer.

SERENA [17] allows router nodes to sleep, while ensuring end-to-end communication in the wireless network. It is a localized and decentralized algorithm assigning time slots to nodes. Any node stays awake only during its slot and the slots assigned to its neighbors, it sleeps the remaining time. In S-MAC protocol [18], it switches periodically among the modes of sleep, wake-up, listen and then returns to sleep. Each active period is of fixed $115 \mathrm{~ms}$ size, with a variable sleep period. And the length of sleep period dictates the duty cycle of S-MAC. B-MAC [19] protocol is a lightweight, configurable MAC that is used as the default MAC for Mica2 motes. It is unscheduled, and it adopts CSMA with duty cycles to conserve energy. It uses clear channel assessment and packet backoffs for channel arbitration, link layer acknowledgement for reliability, and low power listening for low power communication. STEM [20] 
protocol uses two-radio architecture, and it saves power by keeping the data radio sleep until communication is needed. The wakeup radio listens periodically using a low duty cycle, which reduces the energy of idle listening. STEM uses an asynchronous beacon packet in special wakeup channel to wakeup the intended receiver.

By and large, the issue about mitigating total average times of triggering radio transmission throughout node's lifetime is not a concern for these open literatures [7,8,15-20]. Moreover, the EHP has not ever been the major issue for their research target. The wakeup action is basically a process of turning on the radio server and applies to any MAC protocol for a sensor node [20]. It performs initial configuration of the radio, starts the radio and its oscillator, then switches the radio to receive or/and transmit modes, and finally performs the necessary actions. From the viewpoint of power consumption, the cost for powering up the radio is almost the same for all protocols. The difference between various MAC protocols is how long the radio is on after it has been started and how many times the radio is re-started. The latter issue, how to decrease the total average times of triggering radio transmission throughout nodes lifetime, is the target for this research.

A queue threshold, $\mathrm{D}$, is specified in the concept of data aggregation for optimizing power consumption. This threshold could be used to control the average times of turning on the data radio for the buffered data packets. In the "data aggregation" scheme, a sensor node triggers the radio transmission unit, only when the data buffer reaches the threshold value and subsequently conducts the basic process of transmitting packets for a generic senor node. An interesting issue arises: Is it possible to provide a modeling approach to configure the queue threshold D for the IEEE 802.15.4/ZigBee sensor node in terms of optimizing power consumption?

The D-policy M/G/1 queuing model [14] is adopted and the relevant mathematical formulism is established. The D-policy M/G/1 queuing model is expressed in terms of a Petri Net design for qualitative analysis. Moreover, we configure the optimal D for the control of triggering on the radio server of ZRs, especially for the ZRs in the first depth. Our work is the follow-up effort of our conference paper [21]. In contrast to our conference paper, this article provides a more in-depth presentation of the models and describes the implementation of a comprehensive NS2 network simulator. With this network simulator, sensor node administrators and users can make a model of the node to evaluate it with respect to other hardware configurations and application patterns.

\section{A petri net design and mathematical preliminary}

\subsection{Petri net design}

Petri Nets (PNs) are a graphical mathematical modeling tool application to many systems [22]. They are a promising tool for describing and studying information processing systems that are characterized as being concurrent, asynchronous, distributed, parallel, nondeterministic, and/or stochastic. A PN is identified as a particular kind of bipartite directed graph populated by three types of objects. They are places, transitions, and directed arcs connecting places and transitions. A PN is a 5-tuple, $\mathrm{PN}=\{\mathrm{P}, \mathrm{T}, \mathrm{I}$, $\left.\mathrm{O}, \mathrm{M}_{0}\right\}$ [23] where:

$\mathrm{P}=\left\{\mathrm{p}_{1}, \mathrm{p}_{2}, \ldots, \mathrm{p}_{m}\right\}$ is a finite set of places, where $m>0$;

$\mathrm{T}=\left\{\mathrm{t}_{1}, \mathrm{t}_{2}, \ldots, \mathrm{t}_{n}\right\}$ is a finite set of transitions with $\mathrm{P} \cup \mathrm{T} \neq \emptyset$ and $\mathrm{P} \cap \mathrm{T}=\emptyset$, where $n>0$;

$\mathrm{I}: \mathrm{P} \times \mathrm{T} \rightarrow \mathrm{N}$ is an input function that defines a set of directed arcs from $\mathrm{P}$ to $\mathrm{T}$,

where $N=\{0,1,2 \ldots\}$;

$\mathrm{O}: \mathrm{T} \times \mathrm{P} \rightarrow \mathrm{N}$ is an output function that defines a set of directed arcs from $\mathrm{T}$ to $\mathrm{P}$.

$\mathrm{M}_{0}: \mathrm{P} \rightarrow \mathrm{N}$ is the initial marking. 


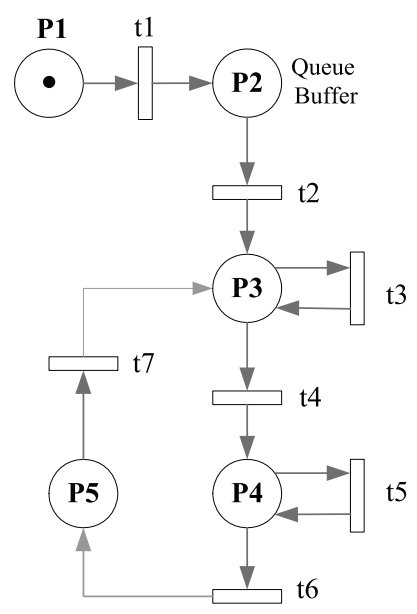

Fig. 1. A petri net design of proposed system.

A transition $t$ is enabled if each input place $p$ of $t$ contains at least the number of tokens equal to the weight of the directed arc connecting $\mathrm{p}$ to $t$. When an enabled transition fires, it removes the tokens from its input places and deposits them on its output places. PN models are suitable to represent the system that characterizes event-driving, choice, concurrency, conflict, and synchronization. In graphical representation, places are drawn as circles, transitions as bars or boxes. In modeling, using the concept of conditions and events, places represent conditions, and transitions represent events. A transition (an event) has a certain number of input and output places representing the pre-conditions and post-conditions of events, respectively. Other detailed properties, analysis and applications on PN can be found in [22].

PN has been widely accepted for modeling of discrete event systems for qualitative analysis [23]. The behavior of many systems can be described in terms of system states and their changes. For the proposed approach, the transmitting function of radio server can be turned on or off dynamically based on the condition of D-policy. With the PN modeling technique, an on/off transition on transmitting function of radio server has been designed and developed to reflect the time-varying, dynamical patterns for qualitative analysis.

Queuing Networks (QNs) and PNs are well-known formalisms for the description and analysis of multifarious systems. PNs are used for qualitative analysis whereas QNs are employed for performance evaluation in a quantitative manner. From a practical viewpoint, system analysis should be pursued with respect to both qualitative and quantitative aspects. We propose an event/condition PN to model the states of Zigbee sensor node and the events causing the state transition in a generic sensor node. Based on the prior system description, the PN-based model of operational flow for a generic Zigbee sensor node is constructed in Fig. 1. The corresponding PN notations for states (places) and transitions (events) are defined in PN_Notation 1 and PN_Notation 2 respectively.

PN_Notation 1 There are seven transitive states (places) for a router node during modeling proposed queue-based system:

P1: A Zigbee sensor node is available

P2: Queue buffer for incoming packets, including self-sensed and relay packets

P3: Radio server is in Idle State; Packet-sending is off

P4: Radio server is in Busy State; Packets are transmitting

P5: A busy cycle has completed; Transmitting function of radio server is off 
PN_Notation 2 Given the set of transitive states (places) $B=\{P 1, P 2, \ldots, P 5\}$, we define the state transitions (events) during modeling proposed system:

t1: Transition ' $\mathrm{t} 1$ ' is enabled when packet arrives in place 'P1'

t2: Transition ' $\mathrm{t} 2$ ' is enabled when packets are ready for transmission service

t3: D-policy condition is not met

t4: D-policy condition is satisfied

t5: Queue buffer is not empty (Exhaustive service)

t6: Exhaustive service has completed and the queue is empty

t7: System state is back to the Idle State

\subsection{Mathematical preliminary}

The mapping relation between queuing theory and our application in ZSN can be envisioned as follows: a "customer" arriving and queued in the queuing system for the server's service represents a "data packet" arriving and queued in the Zigbee device for the radio server's transmission. The size of queue buffer in each ZigBee device is assumed to be large enough to be regarded as infinity. The buffer is modeled as a centralized FCFS queue. The wireless channel is assumed to be error-free. The communication pattern is assumed to be many-to-one, in which a group of sensor nodes at the same depth only communicate to their parent sensor in a one-hop environment. The final destination node could be the $\mathrm{ZC}$, a data sink, or a data cluster.

From the standpoint of queuing theory [22], under the assumptions of the D-policy, it is possible for the server to identify each customer's payload which is his service time. Thus, the idle server starts providing service as soon as the total payload for all customers in the waiting line exceeds $\mathrm{D}$, where the total payload is the sum of each customer's service time. In our application, each customer's payload in queuing theory is equivalently mapped into each arriving packet's payload in sensor node. The payload data implies the contents of a data message that is being transmitted.

It is assumed that packets arrive following a Poisson process with average rate $\lambda$ and the service time by radio server according to a general distribution with first three moments $E[S], E\left[S^{2}\right]$ and $E\left[S^{3}\right]$. Without loss of generality, we consider two major operational states for the radio server of the sensor node: idle and busy, which are equivalent to the monitoring and active states respectively. In general, the idle state is mapped to the lowest value of the radio server power consumption, which can be viewed as the state of turning off the transmitting function of radio server; while being busy state, energy is spent in the front-end amplifier that supplies the power for actual RF transmission for the standard process of medium-contention and the subsequent phase of sending packets. The busy state can be viewed as the state of turning on the radio server.

We study the behavior of a single generic sensor by developing the D-policy M/G/1 queuing model, in which the operating flow for the sensor node can be modeled as shown in Fig. 1. On the transition (event) $t 4$ of Fig. 1, before accumulating D data payload in queue buffer, the function of transmitter in radio server is turned off (place P3: Server in Idle Mode). At the instant of the transition t4 is met, the transmitter is turned on and begins service for transmitting data packets in the buffer exhaustively (place P4: Sever in Busy Mode).

The server is turned off (idle) at the end of each complete period as shown in place P5 of Fig. 1 and is activated again only when the cumulative workload firstly exceeds the fixed value D. In 2008, Wang et al. [14] first developed various system performance measures such as the expected number of customers, the expected length of the turned-off, busy, and breakdown periods in the D-policy M/G/1 
queuing system with server breakdowns. Following the results in expression (28) of Wang [14], the expected number of customers $\left(L_{D}\right)$ in the D-policy M/G/1 queuing system with server breakdowns has the following result:

$$
L_{D}=D+\frac{\lambda \theta_{2}}{2\left(1-\rho_{H}\right)}-\frac{D+\int_{0}^{D} M_{H}(y) d y}{1+M_{H}(D)} .
$$

Here, $M_{H}(D)$ is the renewal function: $M_{H}(y)=\sum_{n=1}^{\infty} F_{H}^{(n)}(y)$, and $F_{H}^{(n)}(D)$ is the n-fold convolution of $\mathrm{F}$ with itself. However, in practical sensor network environment, because it is rather difficult to repair or recharge the sensor modes that are run out of batteries, the breakdown factor would not be taken into account. Hence, For properly fitting our sensor node case, the terms $\rho_{H}, \theta_{2}, \mathbf{M}_{H}(\mathrm{y})$, and $\mathbf{M}_{H}(\mathrm{D})$ are reduced to $\rho, E\left[S^{2}\right], M(y)$, and $M(D)$, respectively. Hence Eq. (1) is simplified to be the following form:

$$
L_{D}=D+\frac{\lambda E\left[S^{2}\right]}{2(1-\rho)}-\frac{D+\int_{0}^{D} M(y) d y}{M(D)+1} .
$$

\subsection{System performance measures}

\subsubsection{Expected length of the busy period and the idle period}

In the ordinary M/G/1 queuing system (i.e., without D-policy), from expressions (5) and (6) of Gakis et al. [24], we obtain the expected length of busy period and idle period, denoted by $\mathrm{B}_{0}$ and $\mathrm{I}_{0}$ respectively, as follows:

$$
E\left[B_{0}\right]=\frac{E[S]}{(1-\rho)} \text { and } E\left[I_{0}\right]=\frac{1}{\lambda}
$$

Then following expressions (30) and (35) of their work, the expected length of the busy period and idle period in the D-policy M/G/1 queuing systems, denoted by $B_{D}$ and $I_{D}$ respectively, can be obtained as follows:

$$
\begin{aligned}
& E\left[B_{D}\right]=E\left[B_{0}\right][M(D)+1]=\frac{E[S][M(D)+1]}{1-\rho} \\
& E\left[I_{D}\right]=E\left[I_{0}\right][M(D)+1]=\frac{[M(D)+1]}{\lambda}
\end{aligned}
$$

\subsubsection{Expected length of the busy cycle}

The busy cycle for D-policy M/G/1 queuing system, denoted by $\Omega_{D}$, is the length from the beginning of the last radio server turned-off (idle) period to the beginning of the next turned-off (idle) period. Since the busy cycle is the sum of the turned-off period $\left(I_{D}\right)$ and the busy period $\left(B_{D}\right)$, we get the following expression from Eqs (3) and (4):

$$
E\left[\Omega_{D}\right]=E\left[B_{D}\right]+\left[I_{D}\right]=\frac{E[S][M(D)+1]}{1-\rho}+\frac{[M(D)+1]}{\lambda}=\frac{[M(D)+1]}{\lambda(1-\rho)}
$$

\subsection{Formation on power consumption function and data simulation}

\subsubsection{Power consumption function}

The total expected power consumption function, $P_{C}(D)$ is developed for the proposed model. Our objective is to establish the closed form of power consumption function in terms of relevant parameters 
and power consumption elements for practical situations. Since there is only one server setup for each busy cycle, it is reasonably assumed that fixed energy consumption is incurred per busy cycle by switching from idle mode to busy mode and vice versa. The sum of these two types of energy waste, called the setup energy consumption factor, is given by $C_{s}$. Let

$\mathrm{C}_{s}=$ setup energy for per busy cycle

$\mathrm{C}_{h}=$ holding power per unit of workload in the system

$\mathrm{C}_{b}=$ power consumption while radio server is in busy state

$\mathrm{C}_{i d}=$ power consumption while radio server is in idle state

By the definitions of each power consumption factors, the power consumption function is given by

$$
P_{C}(D)=\frac{C_{s}}{E\left[\Omega_{D}\right]}+C_{h} L_{D}+C_{b} \frac{E\left[B_{D}\right]}{E\left[\Omega_{D}\right]}+C_{i d} \frac{E\left[I_{D}\right]}{E\left[\Omega_{D}\right]},
$$

where $L_{D}, E\left[B_{D}\right], E\left[I_{D}\right]$ and $E\left[\Omega_{D}\right]$ are given in Eqs (2), (3), (4), and (5) respectively. Putting them into Eq. (6) yields

$$
P_{C}(D)=C_{s} \frac{\lambda(1-\rho)}{M(D)+1}+C_{h}\left[D+\frac{\lambda E\left[S^{2}\right]}{2(1-\rho)}-\frac{D+\int_{0}^{D} M(y) d y}{M(D)+1}\right]+C_{b} \rho+C_{i d}(1-\rho) .
$$

To make notations clearer, it is assumed that the service times $\mathrm{S}$ has a finite $k^{\text {th }}$ moment $\mu_{k}=E\left[S^{k}\right]$ $(k=1,2,3)$. Then we now develop the asymptotic expansions as follows. Suppose that D is sufficiently large compared with E[S]. Following the result of expression (1.46) in [25], the average total power consumption function with reliable server is approximated by:

$$
\begin{aligned}
P_{C}(D) & \doteq\left(\frac{D}{\mu_{1}}+\frac{\mu_{2}}{2 \mu_{1}^{2}}\right)^{-1}\left[C_{s} \lambda(1-\rho)-\frac{C_{h}}{2} A(D)\right]+C_{h} D+\frac{C_{h} \lambda \mu_{2}}{2(1-r h o)}+C_{b} \rho+C_{i d}(1-\rho) \\
& =\frac{1}{A^{\prime}(D)}\left[2 C_{s} \lambda(1-\rho)-C_{h} A(D)\right]+C_{h} D+\frac{C_{h} \lambda \mu_{2}}{2(1-\rho)}+C_{b} \rho+C_{i d}(1-\rho)
\end{aligned}
$$

where $A(D)=\frac{D^{2}}{\mu_{1}}+\frac{D \mu_{2}}{\mu_{1}^{2}}+\frac{\mu_{2}^{2}}{2 \mu_{1}^{3}}-\frac{\mu_{3}}{3 \mu_{1}^{2}}$ and $A^{\prime}(D)=\frac{2 D}{\mu_{1}}+\frac{\mu_{2}}{\mu_{1}^{2}}$.

\subsubsection{Optimization feasibility by numerical validation}

The analytic study of the behavior of the expected power consumption function $P_{C}(D)$ in (8) would have been an arduous task to undertake since the decision variable $D$ appears in an expression which is highly on-linear and complex. For illustrative purpose, it is assumed that packets for sensor node arrive according to a Poisson process with average arrival rate $\lambda$. The service times are independent and identically distributed random variable obeying an arbitrary distribution function $S(t)(t \geqslant 0)$ with first three moments $\mathrm{E}[\mathrm{S}], \mathrm{E}\left[\mathrm{S}^{2}\right]$ and $\mathrm{E}\left[\mathrm{S}^{3}\right]$. All simulations are performed with MATLAB 7.6 on Intel Core 2 Quad CPU (2.4 GHz clock, 2 G RAM). Custom MATLAB scripts are written to simulate the proposed power-saving scheme. Simulations are compared with ordinary M/G/1 queuing case without $D$ policy.

Data Simulation 1:

Let the service time (S) of the radio server has exponential distribution. The first three moments of the service time are given by $\left(\mathrm{E}[\mathrm{S}], \mathrm{E}\left[\mathrm{S}^{2}\right], \mathrm{E}\left[\mathrm{S}^{3}\right]\right)=\left(\mathrm{E}[\mathrm{S}], 2 /(\mathrm{E}[\mathrm{S}])^{2}, 6 /(\mathrm{E}[\mathrm{S}])^{3}\right)=\left(0.05,5 \times 10^{-3}, 7.5 \times\right.$ $\left.10^{-4}\right)$ if the average service time $\mathrm{E}[\mathrm{S}]$ is set to be 0.05 . Other system's parameters are assumed to be as follows:

- average arrival rate of arriving data packets: $\lambda$

- power consumption factors: $\mathrm{C}_{s}=30, \mathrm{C}_{h}=0.2, \mathrm{C}_{b}=50$, and $\mathrm{C}_{i d}=10$. 


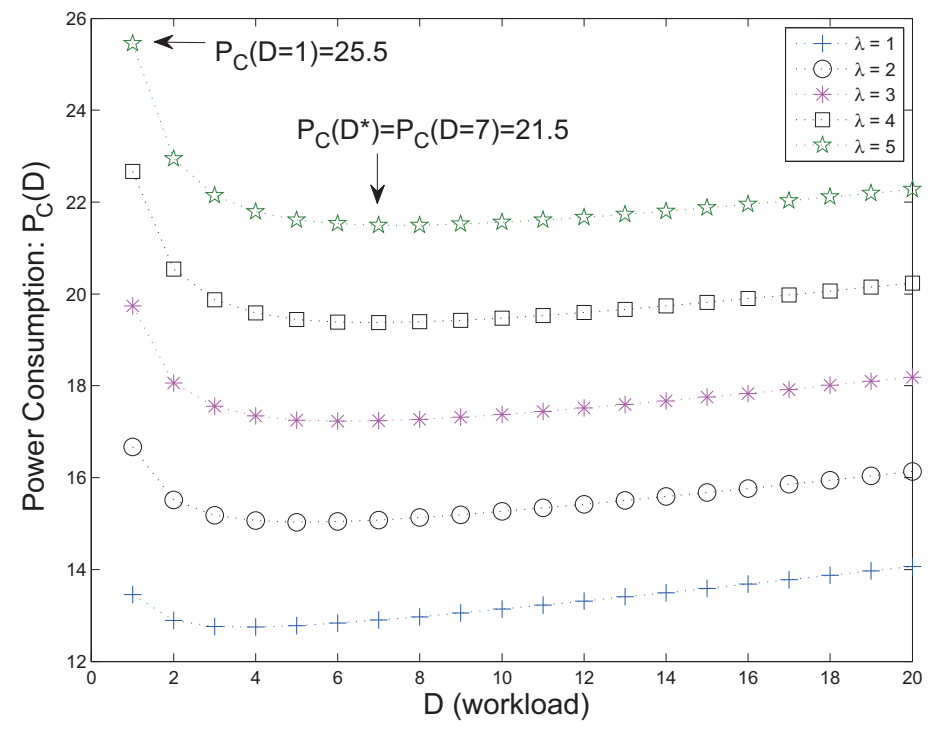

Fig. 2. Power consumption, $\lambda: 1 \sim 5$.

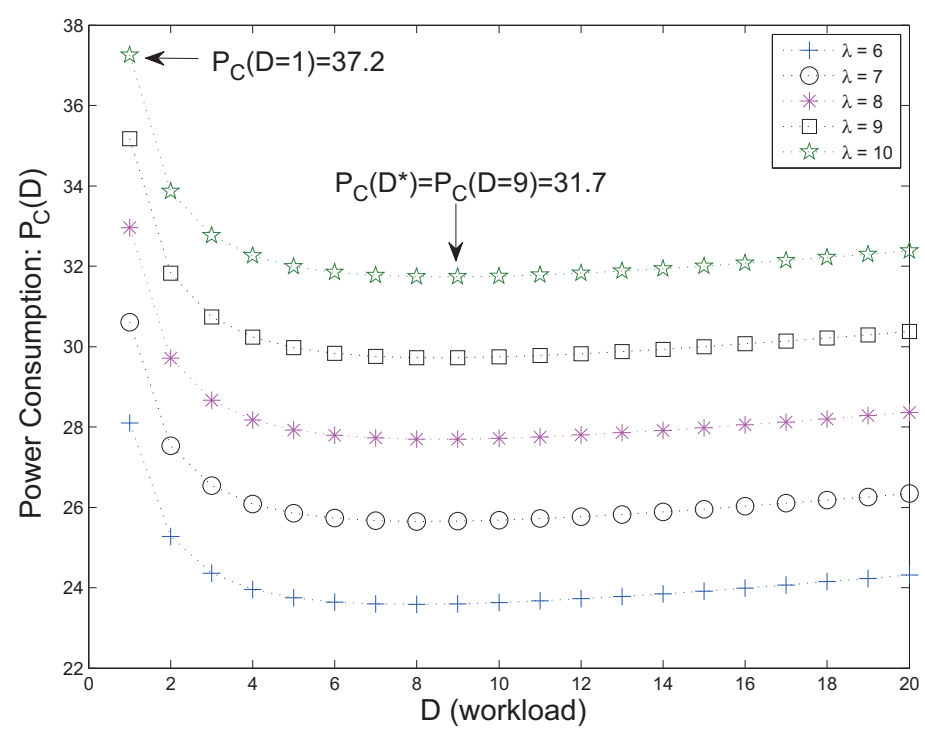

Fig. 3. Power consumption, $\lambda: 6 \sim 10$.

It is noted that the average service rate of radio server is 20 if the average service time $E[S]$ is set to be 0.05. Based on (8), we study the effect of varying different average arrival rate (from 1.0 to 10.0) to obtain the corresponding optimal D value while keeping other parameters constant. Each total expected power consumption function $\mathrm{P}_{C}(\mathrm{D})$ can be simulated based on Eq. (8). Basically, the contour plots provide the best graphical representation of the optimization problem, and also possess a powerful visualization that permits the solutions of the optimization problem by inspection.

We have the graphical results using MATLAB software package as shown in Figs 2 and 3. In these two figures, $P_{C}(D)$ is concave upward by showing a relative low point in power consumption for various $\lambda$ 


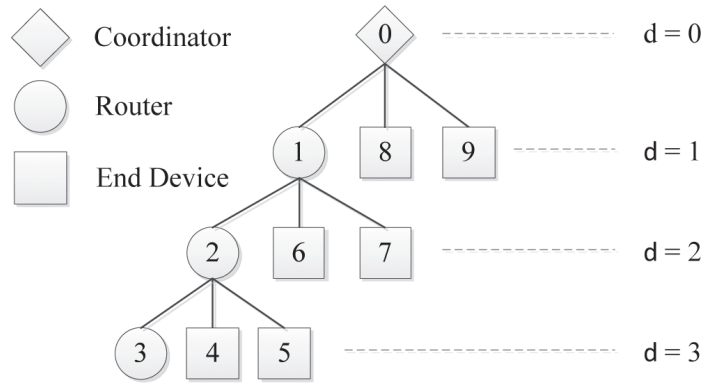

Fig. 4. Addressing assignment for the topological parameter vector $\left[L_{m}, C_{m}, R_{m}\right]=[3,3,1]$.

values from 1 to 5 and from 6 to 10 respectively. For each contour with fixed $\lambda$ value, a local minimal for power consumption emerges and then it reveals the possibility of optimal design. Taking the contour with $\lambda=5$ in Fig. 2 (pentagons) as an example, the optimal power consumption of ZSN, $P_{C}(D *)$, may be improved by an amount of $15.6 \%$ relative to $P_{C}(D=1)$. Similarly, Observing the curve with $\lambda=10$ in Fig. 3 (pentagons), $P_{C}(D *)$ may be improved by an amount of $14.8 \%$ relative to $P_{C}(D=$ 1). Such an improvement phenomenon has strengthened the effectiveness and feasibility of applying the proposed optimization approach into the MAC-based medium-contention process.

\section{Analyzing traffic loads on ZigBee nodes}

The ZigBee coordinator determines the maximum number of children any device, within its network, is allowed. Of these children, a maximum of nwkMaxRouters can be router-capable devices. The remaining devices shall be reserved for end devices. Every device in ZSN has an associated depth that indicates the minimum number of hops a transmitted frame must travel, using only parent-child links, to reach the ZC. According to ZigBee specification, the ZC is at depth 0 , while its descendants have depths 1,2 and so on. Multi-hop networks have a maximum depth that is greater than 1. Distributed Address Assignment Mechanism (DAAM) is the default addressing scheme defined in the Clause 3.6.1.6 of [8].

DAAM's addressing is tree-based in the sense that any subtree has a block of contiguous addresses. A crucial property of DAAM is that a ZC/ZR is capable of locally allocating addresses to its children with the knowledge of its own depth value and the global setting of the topological parameter. The advantage by combining both tree structure and regularity of addressing significantly reduce the energy waste incurred from the establishment and maintaining on routing table like AODV or DSR used in conventional wireless network. Given values for the maximum number of children a parent may have, nwkMaxChildren $(\mathrm{Cm})$, the maximum depth in the network, nwkMaxDepth $(\mathrm{Lm})$, and the maximum number of routers a parent may have as children, nwkMaxRouters (Rm), we may compute the function, $C_{s k i p}(d)$, essentially the size of the address sub-block being distributed by each parent at that depth to its router-capable child devices for a given network depth, $\mathrm{d}$, as follows [8]:

$$
C_{s k i p}(d)= \begin{cases}1+C_{m} \cdot\left(L_{m}-d-1\right), & \text { if } R_{m}=1 \\ \frac{1+C_{m}-R_{m}-C_{m} \cdot R_{m}^{L_{m}-d-1}}{1-R_{m}}, & \text { Otherwise }\end{cases}
$$

It is assumed that all the nodes are deployed in a ZSN which is formed in tree-based topology with the maximum depth $\left(L_{m}\right)$ in the network. Every node in the whole sensor filed is assumed to have an identical sensing data rate $\omega$ to retrieve the environmental or target information. It is also assumed that 


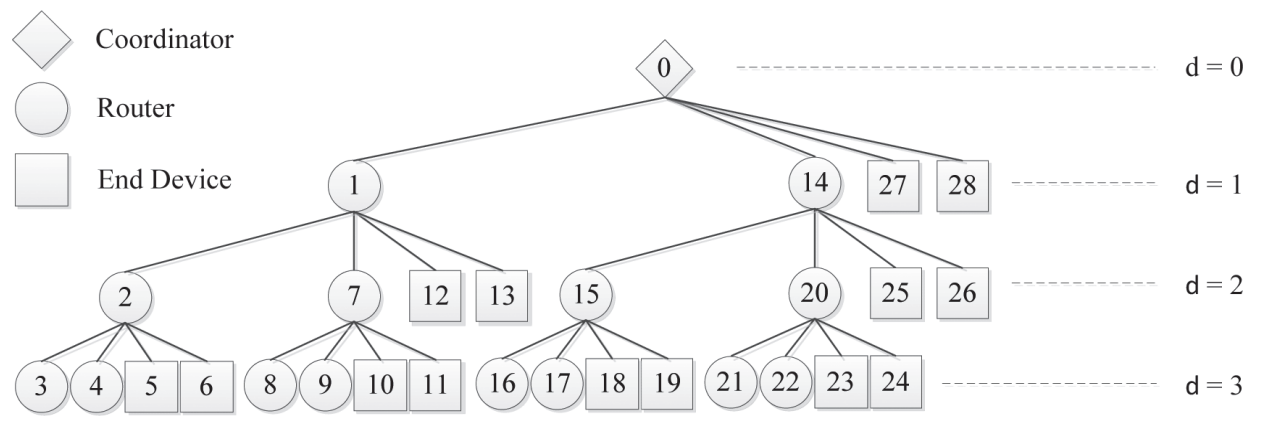

Fig. 5. Addressing assignment for the topological parameter vector $\left[\mathrm{L}_{m}, \mathrm{C}_{m}, \mathrm{R}_{m}\right]=[3,4,2]$.

a packet can traverse each depth using only one hop transmission. For illustrative purpose, we take the topological parameter vectors $\left[L_{m}, C_{m}, R_{m}\right]=[3,3,1]$ and $\left[L_{m}, C_{m}, R_{m}\right]=[3,4,2]$ as examples. Figures 4 and 5 depict their corresponding addressing assignment to ZRs and ZEDs respectively. For each depth, the corresponding size of the address sub-block $C_{\text {skip }}(d)$ is calculated using (9) and shown in vector form: $\left[\mathrm{C}_{\text {skip }}(-1), C_{\text {skip }}(0), C_{\text {skip }}(1), C_{\text {skip }}(2)\right]=[10,7,4,1]$ for Fig. 4 and $\left[\mathrm{C}_{\text {skip }}(-1), C_{\text {skip }}\right.$ (0), $\left.C_{\text {skip }}(1), C_{\text {skip }}(2)\right]=[29,13,5,1]$ for Fig. 5. It is noted that the value of $C_{\text {skip }}(-1)$ implies the total maximal number of nodes which can be deployed under the specific topological parameter set.

Since every ZR has to provide packets-relay service for its children nodes in the tree-based ZSN, the parent nodes closer to the $\mathrm{ZC}$ would have higher average arrival rate on traffic load than those of further away from the ZC. Let the average arrival rate of ZEDs and ZRs at depth $d$ be denoted by $\lambda_{\text {ZED }}(d)$ and $\lambda_{Z R}(d)$ respectively. It is assumed that the basic sensing rate for all nodes except ZC is configured to be $\omega$. Without any forwarding need in each end device, the average arrival rate of each ZED of depth $\mathrm{d}$ is given by

$$
\lambda_{\mathrm{ZED}}(d)=\omega, \text { where } d=1,2, \cdots, L_{m} .
$$

In this article, the full deployment of children nodes for each ZR at every depth is assumed to be our research topology. In other words, it is assumed that $C_{m}$ is the exact number of children a parent has and that $R_{m}$ is the exact number of ZRs a parent has as children. We derive the mathematical expressions of per-node traffic load at depth $\mathrm{d}$ from analyzing $\lambda_{Z R}(d)$ as follows.

Case 1: Firstly, if a parent has exactly only one ZR as child at each depth (i.e., $R_{m}=1$ ), then the average arrival rate of this ZR at depth d includes two sources of which one is its self-sensing data rate $(\omega)$ from this $Z R$ and the other is the relay packets from its descendants. Since there are $\left(L_{m}-\mathrm{d}\right)$ layers (levels) descended from this ZR of depth d, the total number of descendant nodes it has is exactly $\left[C_{m}\left(L_{m}-\mathrm{d}\right)\right]$, each one of which generates average arrival rate of $\omega$. Figure 4 exemplifies addressing assignment for the topological parameter vector $\left[L_{m}, C_{m}\right.$, $\left.R_{m}\right]=[3,3,1]$. Taking the ZR numbered 1 as example in Fig. 4 , this ZR at depth 1 has average arrival rate: $\lambda_{Z R}(1)=\left[1+C_{m}\left(L_{m}-\mathrm{d}\right)\right] \omega=[1+3(3-1)] \omega=7 \omega$.

Case 2: $R_{m}>1$. The term $C_{\text {skip }}(d)$ is the size of the address sub-block distributed by each parent at that depth to its router-capable child devices for a given depth of d. For example, taking $\left[L_{m}\right.$, $\left.C_{m}, R_{m}\right]=[3,4,2], C_{\text {skip }}(0)=13$ is shown in Fig. 5. This implies that there are 13 devices including its descendents from this ZR and itself under full deployment. Hence the average arrival rate of each ZR at depth $d, \lambda_{Z R}(d)$, is equal to $\left[C_{\text {skip }}(d-1) \omega\right]$. 
Combining results of the above two cases, we obtain the average arrival rate of each ZR at depth $\mathrm{d}$ as follows:

$$
\lambda_{\mathrm{ZR}}(d)=\left\{\begin{array}{c}
{\left[1+C_{m} \cdot\left(L_{m}-d\right)\right] \cdot \omega, \text { if } R_{m}=1} \\
C_{s k i p}(d-1) \cdot \omega, \text { if } R_{m}=2,3, \ldots, C_{m}
\end{array}\right.
$$

Next we develop mathematical expressions for the number of ZRs and ZEDs at depth $\mathrm{d}$ which are denoted by $N_{\mathrm{ZR}}(d)$ and $N_{\mathrm{ZED}}(d)$ respectively. Since $R_{m}$ is the exact number of ZRs a parent has as children, the number of ZRs at depth $\mathrm{d}$ is

$$
N_{\mathrm{ZR}}(d)=R_{m}^{d}, \text { where } d=1,2, \cdots, L_{m} .
$$

In Fig. 5, for example, there are 4 ZRs (circles numbered 2, 7, 15, 20) at depth 2, which can be obtained from (12): $N_{\mathrm{ZR}}(2)=(2)^{2}=4$. For the specific case when $R_{m}=1$, expression (12) shows that $N_{Z R}$ $(d)$ is reduced to one, which can be exemplified in Fig. 4 where each depth has only one ZR.

Under the assumption of full deployment in ZSN, every ZR at any depth (except depth 0 ) has fixed $\left(C_{m}-R_{m}\right)$ end devices. To explore the mathematical expression for the number of ZEDs at depth d, $N_{\text {ZED }}(d)$, we first consider the case where $R_{m}$ equals one. It is obvious that $N_{\text {ZED }}(\mathrm{d})=\left(C_{m}-1\right)=$ 2 is exemplified at depth $\mathrm{d}$ in Fig. 4. For $R_{m}>1$, the total number of ZRs of depth d is $R_{m}^{d}$ from (14). Observing the grouping profile between depth 2 and depth 3 in Fig. 5, it is noted that the number of groups descended from parent ZRs of depth $(d-1)$ is $R_{m}^{d} / R_{m}$. In Fig. 5, there are 4 groups at depth 3 , each group of which is descended from a parent ZR at depth 2. Each group has fixed $\left(C_{m}-R_{m}\right)$ numbers of ZEDs and hence the mathematical expression for $N_{\mathrm{ZED}}(d)$ is derived as follows:

$$
N_{\mathrm{ZED}}=\left\{\begin{array}{l}
C_{m}-R_{m}, \text { if } R_{m}=1 \\
R_{m}^{(d-1)} \cdot\left(C_{m}-R_{m}\right), \text { if } R_{m}=2,3, \ldots, C_{m}
\end{array}\right.
$$

Putting (10)-(13) together, the neat closed-form mathematical expression for the total average traffic load for depth d, denoted by $T(d)$, can be obtained as follows:

$$
T(d)=N_{\mathrm{ZR}}(d) \cdot \lambda_{Z R}(d)+N_{\mathrm{ZED}}(d) \cdot \lambda_{\mathrm{ZED}}(d) \text {, where } d=1,2, \cdots, L_{m} .
$$

Basically, it is noted that the average traffic load of depth $d, T(d)$, is composed of self-sensed packets by the ZigBee devices at the current depth and relay packets from descendants. From the standpoint of power consumption, the ZRs undertake the responsibility to forward most of traffic load to their parent ZRs. The ZRs nearer the sink would carry heavier traffic loads than those far away from the sink. We demonstrate such an EHP-like situation quantitatively with the following example.

Example 1: From Fig. 5, the topological parameter vector $\left[\mathrm{L}_{m}, \mathrm{C}_{m}, \mathrm{R}_{m}\right]=[3,4,2]$ with four ZRs in each depth, there are total 3 depths in this ZSN. By applying (9), $\left[\mathrm{C}_{\text {skip }}(0), \mathrm{C}_{\text {skip }}(1), \mathrm{C}_{\text {skip }}(2)\right]=[13$, $5,1]$ for Fig. 5. Using (10)-(14) on depths 3, 2 and 1, the relevant traffic metrics at each depth are calculated and listed in the following vectors:

$$
\begin{aligned}
& \mathrm{d}=3:\left[\mathrm{N}_{Z R}(3), \mathrm{N}_{\mathrm{ZED}}(3), \lambda_{Z R}(3), \lambda_{\mathrm{ZED}}(3), \mathrm{T}(3)\right]=[8,8, \omega, \omega, 16 \omega] . \\
& \mathrm{d}=2:\left[\mathrm{N}_{Z R}(2), \mathrm{N}_{\mathrm{ZED}}(2), \lambda_{Z R}(2), \lambda_{\mathrm{ZED}}(2), \mathrm{T}(2)\right]=[4,4,5 \omega, \omega, 24 \omega] . \\
& \mathrm{d}=1:\left[\mathrm{N}_{Z R}(1), \mathrm{N}_{\mathrm{ZED}}(1), \lambda_{Z R}(1), \lambda_{\mathrm{ZED}}(1), \mathrm{T}(1)\right]=[2,2,13 \omega, \omega, 28 \omega] .
\end{aligned}
$$

Observing the growing profile on average traffic load from depth 3 to depth $2,[\mathrm{~T}(2)-\mathrm{T}(3)]=8 \omega$ is the traffic loads generated by the $2^{\text {nd }}$ depth itself where there are exact eight devices deployed at depth 2 in Fig. 5. And also from depth 2 to depth $1,[\mathrm{~T}(1)-\mathrm{T}(2)]=4 \omega$ is the traffic loads generated by the $1^{\text {st }}$ depth itself where there are exactly four devices deployed at depth 1. For ZRs at intermediate depths, obviously each ZR of depth1 has to transmit 13 times of its self-sensed packets $(\omega)$, which reveals the origin of the EHP and the incurred potential threat on operational lifetime of ZSN. 


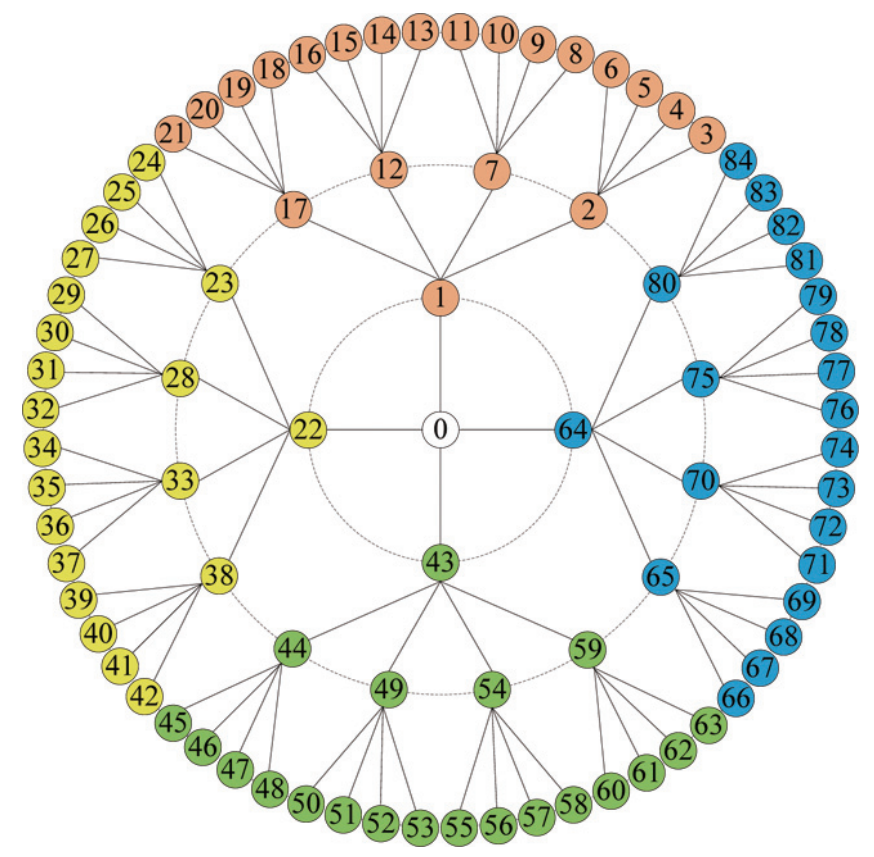

Fig. 6. Topology for the tree-based ZSN.

\section{Optimizing technique on elongating ZSN}

\subsection{Design scenario}

Due to the impact from EHP, the ZRs at the first depth will be destined for a much shorter lifetime compared to the nodes in outer depths, given that all ZRs are equipped with the same battery energy budget. What is even worse, once the ZRs at the first depth are depleted of energy, the sink (ZC) is disconnected from the rest of the ZigBee sensor network. Moreover, the valuable and residual battery energy resources stored in the outer nodes will be useless and wasted eventually. For illustrative purpose, we exemplify a topological parameter vector with $\left[L_{m}, C_{m}, R_{m}\right]=[3,4,4]$. As shown in Fig. 6, ZigBee devices are configured to be all ZRs. For each depth, the corresponding size of the address sub-block $C_{\text {skip }}(d)$ are calculated using (9) and shown in vector form: [ $C_{\text {skip }}(-1), C_{\text {skip }}(0), C_{\text {skip }}(1), C_{\text {skip }}$ (2)] $=[85,21,5,1]$. Figure 6 depicts the conceptual topology where a solid line between two nodes indicates that the nodes are within direct transmission range of each other.

Figure 6 demonstrates the scenario deployed in a manner of concentric pattern in practical situation for the conventional tree-based ZSN because of the limited transmission rage in radio server for ZigBee device. In practical deployment scenario, it is reasonably assumed that children nodes should be deployed around their unique parent node because the limitation of one-hop transmission range. There are four groups of nodes in different colors (brown, yellow, green and blue) which depict four corresponding braches originated from ZRs numbered 1, 22, 43, and 64 respectively. The dash-line circle traversing nodes (1), (22), (43) and (4) depicts the node distribution at the depth of 1 , while the next second larger dash-line circle traversing 12 nodes shows the node distribution at the depth of 2 .

If the sensor node's sensing rate is tuned to be $\omega$, the average traffic load for the node, $\lambda_{Z R}(3)$, in the outermost shell contains only its sensed data packets, and thus $\lambda_{Z R}(3)$ is equal to $\omega$ due to no relay-data 


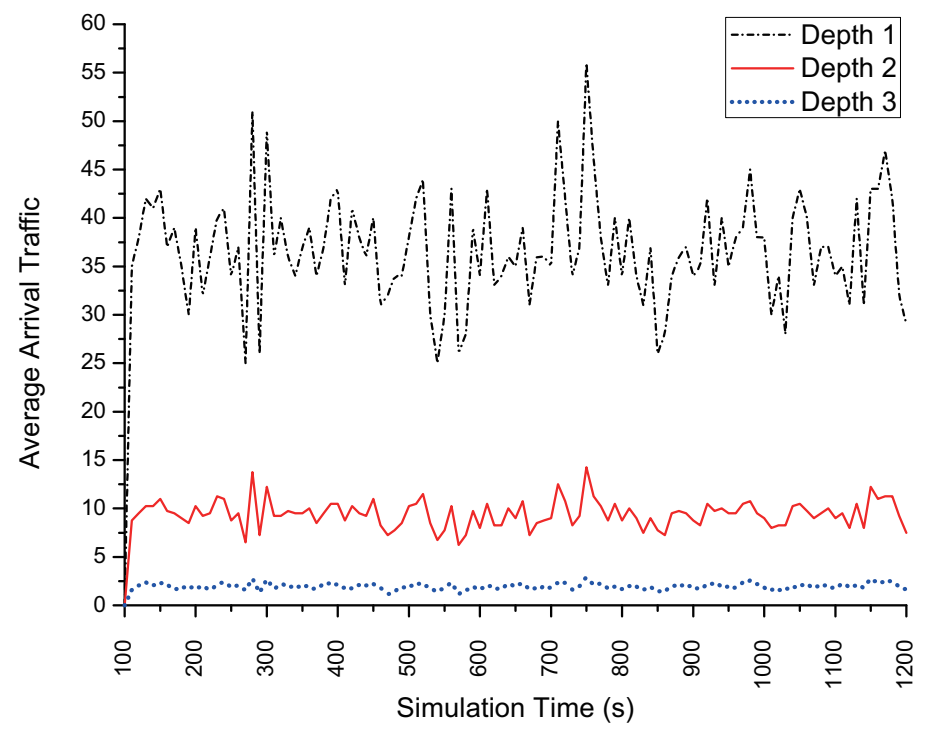

Fig. 7. Average arrival traffic measured $(\omega=0.2)$.

load. In Fig. 6, we calculate the average traffic load using expressions (11), (12) and (14) and yield the following average traffic load in terms of vectors for depth 1 and 2 respectively: $\left[\mathrm{N}_{Z_{R}}(1), \lambda_{Z R}(1), \mathrm{T}(1)\right]$ $=[4,21 \omega, 84 \omega]$ and $\left[\mathrm{N}_{Z R}(2), \lambda_{Z R}(2), \mathrm{T}(2)\right]=[12,5 \omega, 60 \omega]$. It is noted that $\lambda_{Z R}(1) / \lambda_{Z R}(2)=4.2$ and $\lambda_{Z R}(1) / \lambda_{Z R}(3)=21$ which the average traffic load for each ZR at the innermost shell is twentyone times larger than that of $Z R$ at the outermost shell. From the standpoint of power consumption, the operational lifetime for ZR in the innermost shell is also twenty-one times shorter than that of ZR in the outermost shell. This reveals the unavoidable EHP which deteriorates the ZSN lifetime dramatically due to the unbalanced energy depletion.

With little management cost on tuning sensor node's sensing rate, a fixed data sensing rate $(\omega)$ is firstly considered. Having a fixed $\omega$, we can obtain the corresponding average traffic load (average arrival rate) for ZRs in each shell from (11). Applying (8), the average power consumption patterns that correspond to ZRs in each shell can be found out, and analyzed for further optimization. Because of no relay-data need, the ZigBee devices in the outermost shell has the smallest average arrival rate $(\omega)$, and we use it as the base for the normalized average arrival rates for the nodes in inner shells. That is, the average traffic load for ZRs in other shells can be expressed in terms of $\omega$. Taking the topological parameter vector $\left[\mathrm{L}_{m}, \mathrm{C}_{m}, \mathrm{R}_{m}\right]=[3,4,4]$ as shown in Fig. 6, which there are three shells for this ZSN. The average traffic load (average arrival rate) for ZRs in each shell can be computed as the vector: $\left[\lambda_{Z R}(3), \lambda_{Z R}(2)\right.$, $\left.\lambda_{Z R}(1)\right]=[\omega, 5 \omega, 21 \omega]$. Observing the mathematical expression in (8), in additional to the parameter $\mathrm{D}$, the average power consumption pattern, $\mathrm{P}_{C}(\mathrm{D})$, is also a function of $\lambda$ (i.e., $\lambda_{Z R}$ ).

\subsection{Network simulation and performance}

To evaluate and verify the proposed queued-based approach, simulation experiments are conducted in this subsection using the NS2 network simulator [26]. Since the nodes in innermost shell will have a quite larger amount of power consumptions compared to the nodes in outer shells, the lifetime of sensor network is primarily dominated by lifetimes of nodes in innermost shell. Without loss of generality, the planar network topology for tree-based ZSN shown in Fig. 6 is considered. All wireless sensor nodes 


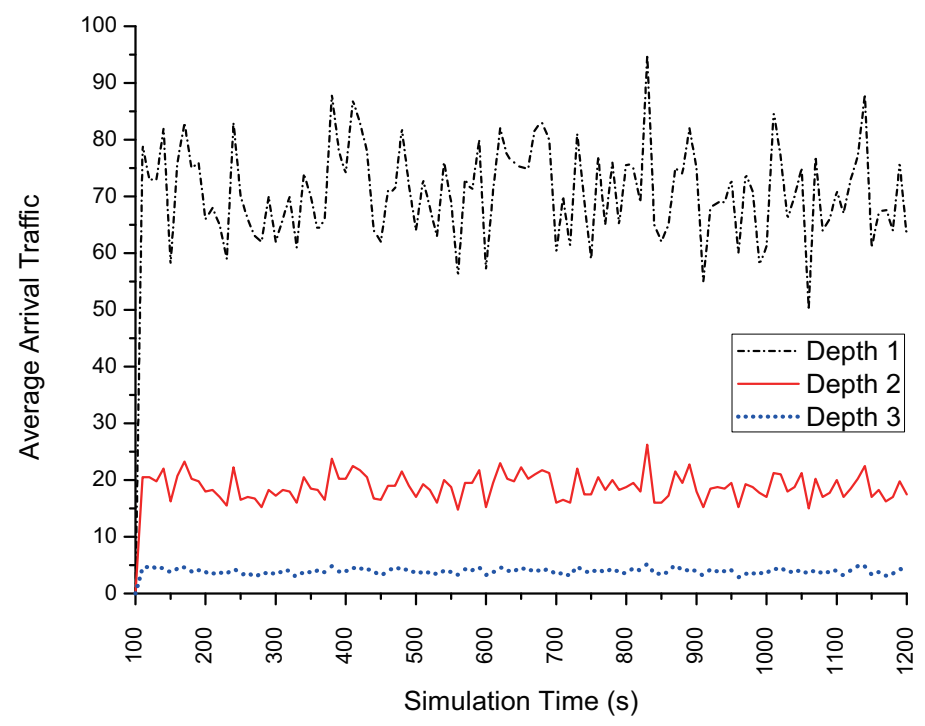

Fig. 8. Average arrival traffic measured ( $\omega 0.4)$.

transmit packets using wireless radios with a bandwidth $250 \mathrm{Kbps}$, and the sources use UDP as the transport protocol. Theoretically, the relative ratio for the average arrival traffic for three depths is: $\lambda_{Z R}$ (3): $\lambda_{Z R}(2): \lambda_{Z R}(1)=1: 5: 21$ because only the ZRs in the two innermost shells of ZSN have the burden to relay packets.

Firstly, focusing on the traffic load analysis firstly, Figs 7 and 8 show the average arrival traffic (in measured packets) per node at each depth in Fig. 6 for simulation duration of 1200 seconds. In Fig. 7, the sensor node's base sensing rate $(\omega)$ is set to be 0.2 , and the number of packets is counted every 10 seconds. The relative ratio measured for the average arrival traffic for three depths is: $\lambda_{Z R}(3): \lambda_{Z R}(2): \lambda_{Z R}(1)=$ 1.0:4.82:18.75, which approach the theoretical ratio (1:5:21) quite well. In Fig. 8, the sensor node's base sensing rate $(\omega)$ is configured to be 0.4 , and the relative ratio is exemplified by an amount of $\lambda_{Z R}(3): \lambda_{Z R}$ (2): $\lambda_{Z R}(1)=1.0: 4.81: 18.03$, which is close to the theoretical ratio as well.

Secondly, to mitigate the direct perturbation from different system parameters settings, relative improvement level is introduced for power consumption evaluation. The improvement degree of power consumption due to the proposed queuing approach may be evaluated by the following equation:

$$
\text { Power Consumption Improvement Factor }(\mathrm{PCIF})=\frac{P_{C, \text { Ordinary }}-P_{C}(D)}{P_{C, \text { Ordinary }}} \times 100 \%
$$

Where $P_{C, \text { Ordinary }}$ represents the power consumption of an ordinary M/G/1 system without the Dpolicy, whereas $P_{C}(D)$ is defined in Eq. (8). In other words, the term $P_{C, \text { Ordinary }}$ can be regarded as the power consumption of a generic ZSN on the IEEE 802.15.4/ZigBee sensor network.

All wireless sensor nodes transmit packets using wireless radios with a bandwidth $250 \mathrm{Kbps}$, and the sources use UDP as the transport protocol. The size of application layer is configured to be 100 bytes per packet. In terms of energy consumption, we adopt power consumption factors listed in Data Simulation 1 in Section 4. Each simulation is run for 3600 time units may provide us hour long traces. The simulation results are conducted by varying both average arrival rate $(\lambda)$ of data packets and control parameter value D. Only the ZRs in the two innermost shells of ZSN are plotted because the ZigBee devices in the outermost shell have no burden of relay packets which the power consumption would be 


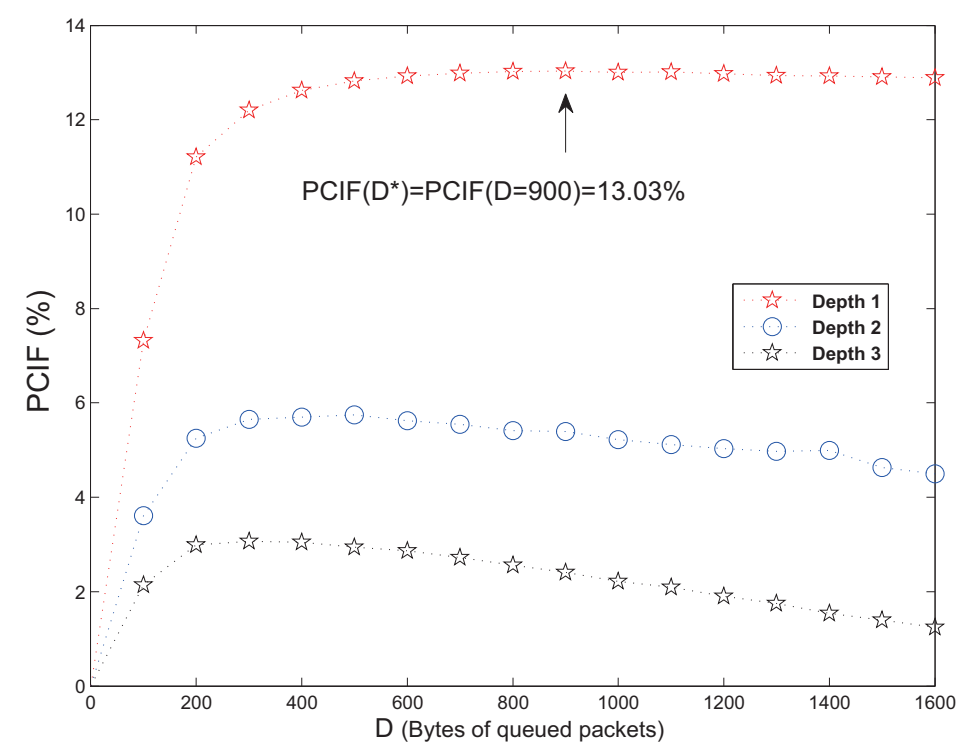

Fig. 9. PCIF curves with $\omega=0.1$.

the smallest one. Each data point in Figs 9 and 10 is the average of 100 runs for each condition with the same topology. The network simulation results are shown in Figs 9 and 10 with base sensing rate $(\omega)$ set at 0.1 and 0.3 respectively.

Basically, these two graphs are all concave downward, which is in agreement with the geometrical implication of contours in term of PCIF metric. The downward-concavity characteristics shown on each curve bring important and convincible information that the optimality approach by the proposed queuebased scheme is effective and feasible. The effectiveness and feasibility of the proposed scheme has been verified by the downward concavity on each PCIF curves in Figs 9 and 10. Moreover, the improvement degree on power consumption and lifetime can be verified by the NS2 simulation results. Let us take the curves of depth 1 (red stars) in Figs 9 and 10 as examples, the optimal PCIF of sensor nodes in the innermost shell may be improved by an amount of $13.03 \%\left(D^{*}=900\right)$ and $12.72 \%\left(D^{*}=800\right)$ respectively. Hence the lifetime prolongation for ZSN may be achieved and the threat to lifetime security may also be alleviated significantly.

\subsection{Sensitivity analysis on system power elements}

Examining $P_{C}(D)$ in Eq. (8), the set of system power elements $\left(C_{s}, C_{h}, C_{b}, C_{i d}\right)$ have some rudimental influences on power consumption. To provide reference data on how they affect power consumption function, numerical illustrations have been conducted on sensitivity analysis based on changes in multifarious sets of system power elements. To show the varying tendency on PCIF for each power element clearly, only one power element is varied each time while keeping other three constant. The distributions of service time and average arrival rates are assumed to be the same as those in Data Simulation 1 except choosing $\omega=0.3$ and the traffic load of node at the first depth is $\lambda_{Z R}(1)=3.9$. The set $\left(C_{s}, C_{h}, C_{b}\right.$, $\left.C_{i d}\right)=(30,0.2,50,10)$ is set to be the baseline set for our numerical investigations.

Firstly, Table 1 shows that optimal $\mathrm{D}^{*}$ increases gradually as $\mathrm{C}_{s}$ increases. It also reveals that PCIF has some significant improvement as $\mathrm{C}_{s}$ increases. Logically, the proposed queue-based power-saving technique can be used to alleviate the total average times of triggering the radio server, and the power 
Table 1

Optimal D and PCIF values for variables $C_{s}$

\begin{tabular}{ccc}
\hline$\left(\mathrm{C}_{s}, \mathrm{C}_{h}, \mathrm{C}_{b}, \mathrm{C}_{i d}\right)$ & $\mathrm{D}^{*}$ & $\mathrm{PCIF}(\%)$ \\
\hline$(15,0.2,50,10)$ & 5 & 6.83 \\
$(20,0.2,50,10)$ & 6 & 9.44 \\
$(25,0.2,50,10)$ & 6 & 11.97 \\
$(30,0.2,50,10)$ & 7 & $\underline{14.37}$ \\
$(35,0.2,50,10)$ & 7 & 16.70 \\
$(40,0.2,50,10)$ & 8 & 18.85 \\
$(50,0.2,50,10)$ & 9 & 22.89 \\
\hline
\end{tabular}

Table 2

Optimal D and PCIF values for variables $C_{h}$

\begin{tabular}{ccc}
\hline$\left(\mathrm{C}_{s}, \mathrm{C}_{h}, \mathrm{C}_{b}, \mathrm{C}_{i d}\right)$ & $\mathrm{D}^{*}$ & $\mathrm{PCIF}(\%)$ \\
\hline$(30,0.05,50,10)$ & 14 & 17.15 \\
$(30,0.07,50,10)$ & 12 & 16.62 \\
$(30,0.1,50,10)$ & 10 & 15.97 \\
$(30,0.2,50,10)$ & 7 & $\underline{14.37}$ \\
$(30,0.3,50,10)$ & 6 & 13.18 \\
$(30,0.4,50,10)$ & 5 & 12.27 \\
$(30,0.5,50,10)$ & 4 & 11.42 \\
\hline
\end{tabular}

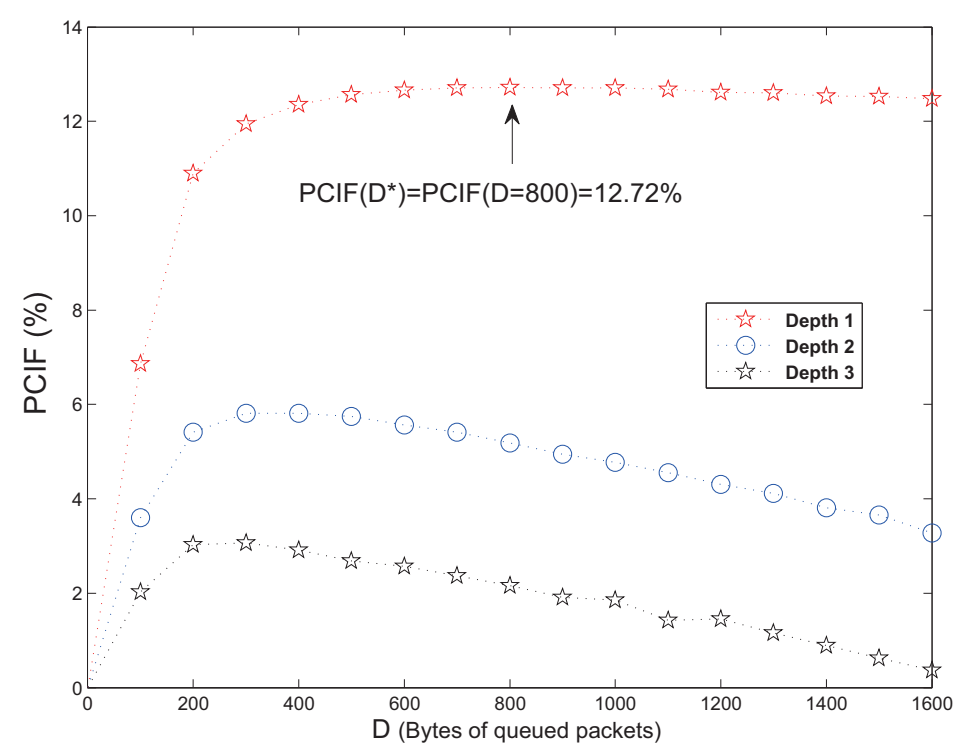

Fig. 10. PCIF curves with $\omega=0.3$.

element $\mathrm{C}_{s}$ implies fixed energy consumption incurred per busy cycle by switching from idle mode to busy mode and vice versa. Hence the transitional energy waste between idle mode and busy mode can therefore be reduced significantly as $\mathrm{C}_{s}$ becomes larger (bottom rows in Table 1). On the other hand, the first four rows in Table 1 display that PCIF becomes deteriorated in a hasty manner (from $14.37 \%$ down to $6.83 \%$ ) as $\mathrm{C}_{s}$ is decreased from 30 to 15 in step of 5. As $\mathrm{C}_{s}$ gets smaller promptly, it seems reasonable that the effect on transitional energy saving would become less influential in the total average power consumption accordingly, and then the corresponding PCIF value would be lowered.

Secondly, how about the impact profile is for the power element $C_{h}$ on PCIF? Table 2 displays that PCIF and optimal D* can be increased slightly as $C_{h}$ decreases. Basically, the total average holding power is proportional to workload (D). When $C_{h}$ increases, the total average holding power for the queued packets would get larger and the corresponding PCIF would become worse associated with smaller D values.

For power consumption of radio server in busy state $\left(C_{b}\right)$, Table 3 demonstrates that PCIF can be increased as $C_{b}$ decreases, while optimal $\mathrm{D} *$ is same as that of the baseline set as implied by Eq. (8). With a similar situation in Table 2, the last row in Table 3 shows that PCIF becomes deteriorated as well (from $14.37 \%$ down to $11.40 \%$ ) as $\mathrm{C}_{b}$ is increased by 1.6 times of the baseline value. The point to such a gradient arises from the increase of $C_{b}$ values (from 50 to 80), which increases the total 
F.-C. Jiang et al. / Toward green sensor field by optimizing power efficiency using D-policy M/G/l queuing systems

Table 3

Optimal D and PCIF values for variables $C_{b}$

\begin{tabular}{ccc}
\hline$\left(C_{s}, C_{h}, C_{b}, C_{i d}\right)$ & $D *$ & $\mathrm{PCIF}(\%)$ \\
\hline$(30,0.2,50,7)$ & 7 & 16.11 \\
$(30,0.2,50,8)$ & 7 & 15.49 \\
$(30,0.2,50,9)$ & 7 & 14.91 \\
$(30,0.2,50,10)$ & 7 & $\underline{14.37}$ \\
$(30,0.2,50,11)$ & 7 & 13.88 \\
$(30,0.2,50,12)$ & 7 & 13.41 \\
$(30,0.2,50,13)$ & 7 & 12.97 \\
\hline
\end{tabular}

Table 4

Optimal D and PCIF values for variables $C_{i d}$

\begin{tabular}{ccc}
\hline$\left(C_{s}, C_{h}, C_{b}, C_{i d}\right)$ & $\mathrm{D}^{*}$ & $\mathrm{PCIF}(\%)$ \\
\hline$(30,0.2,35,10)$ & 7 & 16.53 \\
$(30,0.2,40,10)$ & 7 & 15.75 \\
$(30,0.2,45,10)$ & 7 & 15.03 \\
$(30,0.2,50,10)$ & 7 & 14.37 \\
$(30,0.2,60,10)$ & 7 & 13.22 \\
$(30,0.2,70,10)$ & 7 & 12.24 \\
$(30,0.2,80,10)$ & 7 & 11.40 \\
\hline
\end{tabular}

average power consumption significantly. Hence, the effect from transitional energy saving become less influential in the total average power consumption and then the corresponding PCIF values become lowered inevitably.

Theoretically, the proposed queuing model can mitigate the total average times of transition switching from one mode to another. Observing data in Tables 1 and 3, it indicates that our queue-based approach is more effective in power/energy patterns when the transitional energy can occupy higher proportion of total average power/energy consumption. Shih et al. [27] have shown that the transitional energy when switching from one mode to another significantly impacts the total power consumption. Their work has also demonstrated that as packet size is reduced, the energy consumption is dominated by the startup transient and not by the active transit time. In additional to increasing the data size transmitted in a burst, our queue-based approach can also be used to alleviate such a problem by way of reducing total average times of switching modes. Finally for power element $\left(C_{i d}\right)$, PCIF obtains a little improvement as $C_{i d}$ increases, while optimal D* is same as that of the baseline set as shown in Table 4.

\section{Conclusion}

Toward the formation of green sensor field, the forwarding power consumption is inevitably ubiquitous in most of parent-children links for tree-based ZSNs. The energy hole problem (EHP) then appears to be a security threat to the lifetime of ZSN due to such an unbalanced energy depletion. We concentrate on prolonging the lifetime of ZRs at the first depth by alleviating power consumption using D-policy M/G/1 queuing model. On qualitative analysis, a Petri Net design is provided for in-depth understanding system profile. On quantitative viewpoint, we construct the mathematical power model of a generic sensor node platform, and there exists an optimal queue number (D) that minimizes power consumption in sensor node. In this article, we have provided and analyzed the theoretical aspects of the queue-based powersaving technique which reveals the feasibility of reducing power consumption foe sensor nodes. And also we analyze the average traffic load on ZRs and ZEDs at each depth in terms of neat closed-form mathematical expressions.

With little or no extra management cost, the proposed queue-based approach can be expanded and applied to increase the average lifetime on the ZRs in the dominant shell of the sensor network. To evaluate the proposed design scheme for green sensor field, MATLAB-based simulation results demonstrate that a significant improvement level on power consumption can be achieved. The simulation results using NS2 simulator show that the network lifetime may be prolonged by an amount of at least $12 \%$ due to the saving on average power consumption for the ZRs of depth 1. Hence the proposed powersaving approach indeed provides a feasibly cost-efficient technique to enhance the longevity of the IEEE 802.15.4/ZigBee sensor network and to green the sensor network as well. 


\section{Acknowledgments}

This work was supported in part by the National Science Council, Taiwan ROC, under grant numbers NSC 101-2218-E-029-004, NSC 101-2221-E-029-014, and NSC 101-2622-E-029-008-CC3. This work also supported in part by Tunghai University, Taiwan ROC, under grant number GREEnS 04-2.

\section{References}

[1] I. F. Akyilidiz, W. Su, Y. Sankarasubramaniam and E. Cayirci, Wireless sensor networks: A survey, Computer Networks 38(4) (2002), 393-422.

[2] I. Doha, J. Limb and K. Chaea, Distributed authentication mechanism for secure channel establishment in ubiquitous medical sensor networks, Mobile Information Systems 7 (2011), 189-200.

[3] S. Gupta, S. Verma, G.S. Tomar and R.K. Abrol, Intelligent industrial data acquisition and energy monitoring using wireless sensor networks, International Journal of Grid and High Performance Computing 2(3) (2010), 44-59.

[4] I. You and T. Hara, Mobile and wireless networks, Mobile Information Systems 6(1) (2010), 1-3.

[5] S. Murugesan, Harnessing green IT: Principles and practices, IT Pro. January/February 2008, pp. 24-33.

[6] P. Morreale, F. Qi, and P. Croft, A green wireless sensor network for environmental monitoring and risk identification, International Journal of Sensor Networks 10(1/2) (2011), 73-82.

[7] IEEE 802.15.4 Standard (2003). Part 15.4: Wireless Medium Access Control (MAC) and Physical Layer (PHY) Specifications for Low-Rate Wireless Personal Area Networks. IEEE-SA Standards Board, 2003.

[8] Zigbee Specification 2006. Zigbee Alliance. URL: http://www.zigbee.org.

[9] P. Baronti, P. Pillai, V.W.C. Chook, S. Chessa, A. Gotta and Y.F. Hu, Wireless sensor networks: A survey on the state of the art and the 802.15.4 and ZigBee standards, Computer Communications 30(7) (2007), 1655-1695.

[10] Y. Yu, B. Krishnamachari and V. Prasanna, Energy-latency tradeoffs for data gathering in wireless sensor networks, in: Proc. of the IEEE INFOCOM, March 2004, pp. 244-255.

[11] J. Li and P. Mohapatra, An analytical model on the energy hole problem in many-to-one sensor networks, in: Proc. of IEEE VTC, Dallas, TX, Fall 2005.

[12] J. Lian, K, Naik and G.B. Agnew, Data capacity improvement of wireless sensor networks using non-uniform sensor distribution, International Journal of Distributed Sensor Networks 2(2) (2006), 121-145.

[13] A. Wadaa, S. Olariu, L. Wilson, M. Eltoweissy and K. Jones, Training a sensor networks, Mobile Networks and Applications 10 (2005), 151-168.

[14] K.-H. Wang, C.-C. Kuo and J.-C. Ke, Optimal control of the D-Policy M/G/1 queuing system with server breakdowns, American Journal of Applied Science 5(5) (2008), 565-573.

[15] S. Croce, F. Marcelloni and M. Vecchio, Reducing power consumption in wireless sensor networks using a novel approach to data aggregation, The Computer Journal 51(2) (2008), 227-239.

[16] IEEE, Wireless LAN Medium Control (MAC) and Physical Layer (PHY) Specification, IEEE Std. 802.11-1999 edition (R2003).

[17] S. Mahfoudh and P. Minet, Maximization of energy efficiency in wireless ad hoc and sensor networks with SERENA, Mobile Information Systems 5 (2009), 33-52.

[18] W. Ye, J. Heidemann and D. Estrin, An energy efficient MAC Protocol for wireless sensor networks, in: Proc. of the IEEE INFOCOM, 2002, pp. 1567-1576.

[19] J. Polastre, J. Hill and D. Culler, Versatile low power media for wireless sensor networks, in: Proc. of ACM SenSys, 2004, pp. 3-5.

[20] C. Schurgers, V. Tsiatsis and M. Srivastava, STEM: topology management for energy efficient sensor networks, IEEE Aerospace Conference Proceedings 3 (2002), 3-1099 3-1108.

[21] F.-J. Jiang, C.-T. Yang, C.-H. Hsu, K.-H. Wang and Y.-J. Chiang, Longevity enhancement technique on IEEE 802.15.4/ZigBee sensor network using D-Policy M/G/1 queuing model, in: Proc. of the $14^{\text {th }}$ IEEE International Conference on Computational Science and Engineering (CSE2011), Dalian, China, 2011.

[22] T. Murata, Petri Nets: Properties, analysis and applications, Proceedings of The IEEE 77(4) (1989), 541-580.

[23] J.-S. Lee and P.-L. Hsu, Implementation of a remote hierarchical supervision system using Petri Nets and agent technology, IEEE Transactions of Systems, MAN, and Cybernetics - Part C: Applications and Reviews 37(1) (2007), 77-85.

[24] K.G. Gakis, H.K. Rhee and B.D. Sivazlian, Distribution and the first moments of the busy and idle periods in controllable M/G/1 queuing models with simple and dyadic policies, Stochastic Analysis and Applications 13 (1995), 47-81.

[25] H.C. Tijms, Stochastic Modeling and Analysis: A Computational Approach. John Wiley and Sons, New York, 1986.

[26] The network simulator—ns2. URL: http://nsnam.isi.edu/nsnam/index.php/Main_Page. 
[27] E. Shih, S. Cho, F.S. Lee, B.H. Calhoun and A. Chandrakasan, Design considerations for energy-efficient radios in wireless microsensor networks, Journal of VLSI Signal Processing 37 (2004), 77-94.

Fuu-Cheng Jiang, Ph.D. Currently, he is with the department of computer science at Tunghai University in Taiwan. His research interests include network modeling, cloud computing, wireless networks and simulation. Dr. Jiang was the recipient of the Best Paper Award at the $5^{\text {th }}$ International Conference on Future Information Technology 2010 (FutureTech2010), which ranked his paper first among the 201 submittals. He has served the TPC of the ICCCT 2011-2012 International Conference, BWCCA2010, IEEE CloudCom 2012 and CSE2011 Session Chair. Moreover, he served as journal reviewer of the Computer Journal, Ad Hoc Networks and the International Journal of Communication Systems.

Hsiang-Wei Wu received a B.S. degree from the Department of Electronic Engineering at National Chin-Yi University of Technology in 2002 and a M.S. degree from the Department of Electronic Engineering at Feng Chia University in 2004 respectively. He is a Ph.D. student in the Department of Computer Science and Engineering at the National Chung-Hsing University of Taiwan. Currently, his current interests include the wireless network, network simulation and cloud computing.

Fang-Yie Leu received his B.S., M.S. and Ph.D. degrees from National Taiwan University of Science and Technology, Taiwan, in 1983, 1986 and 1991, respectively, and another M.S. degree from Knowledge System Institute, USA, in 1990. His research interests include wireless communication, network security, Grid applications and Chinese natural language processing. He is currently a professor of Tunghai University, Taiwan, the director of database and network security laboratory of the University, the workshop chair of MCNCS and CWECS workshops, and the editorial board member of several international journals. He is also a member of IEEE Computer Society.

Chao-Tung Yang is a Professor of Computer Science at Tunghai University in Taiwan. He received the PhD in Computer Science from National Chiao Tung University in July 1996. In August 2001, he joined the Faculty of the Department of Computer Science at Tunghai University. He is serving in a number of journal editorial boards, including International Journal of Communication Systems, Journal of Cloud Computing, "Grid Computing, Applications and Technology" Special Issue of Journal of Supercomputing, and "Grid and Cloud Computing" Special Issue of International Journal of Ad Hoc and Ubiquitous Computing. Dr. Yang has published more than 200 papers in journals, book chapters and conference proceedings. His present research interests are in cloud and grid computing, parallel computing, and multicore programming. He is a member of the IEEE Computer Society and ACM. 

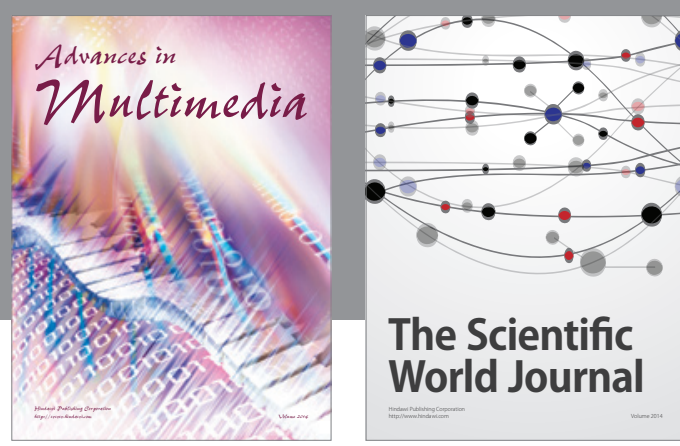

The Scientific World Journal
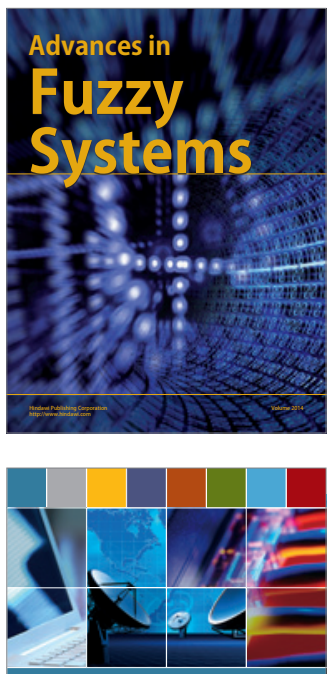

Computer Networks and Communications
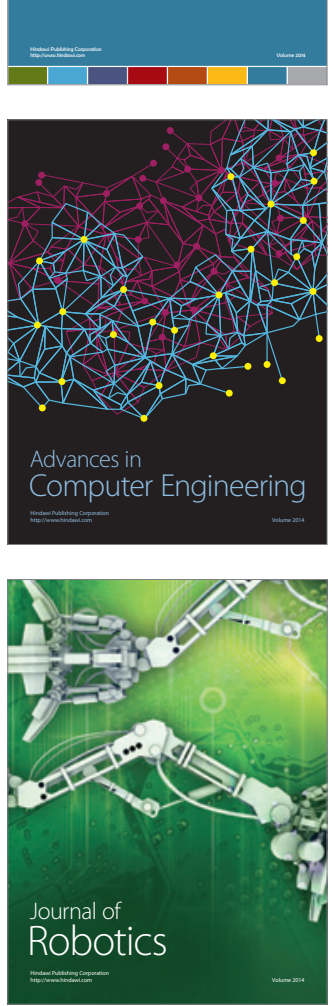
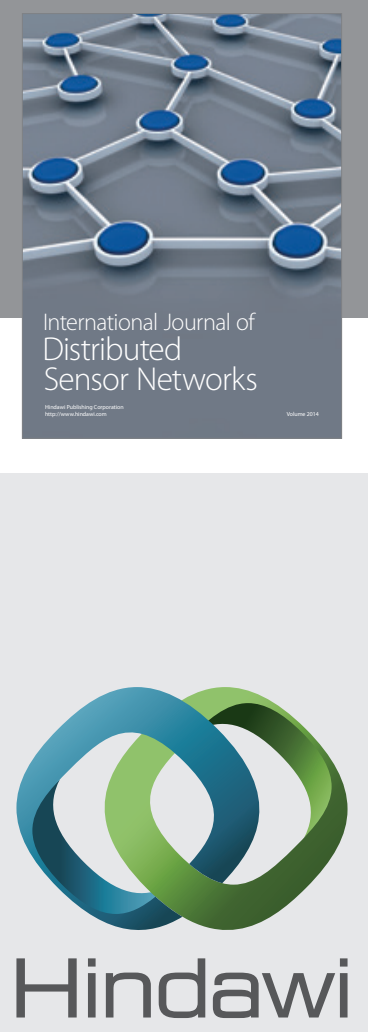

Submit your manuscripts at

http://www.hindawi.com
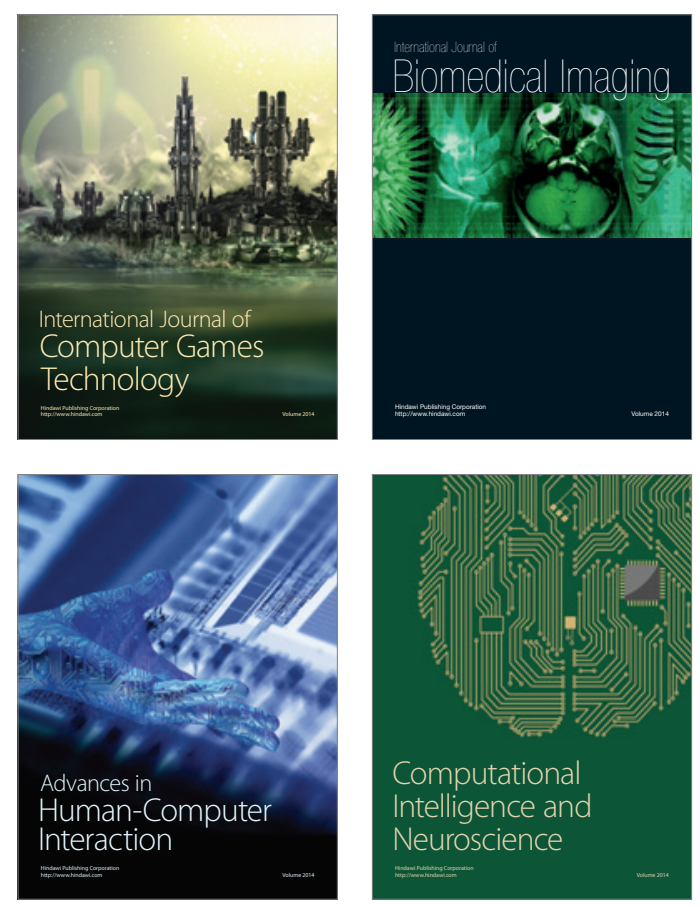
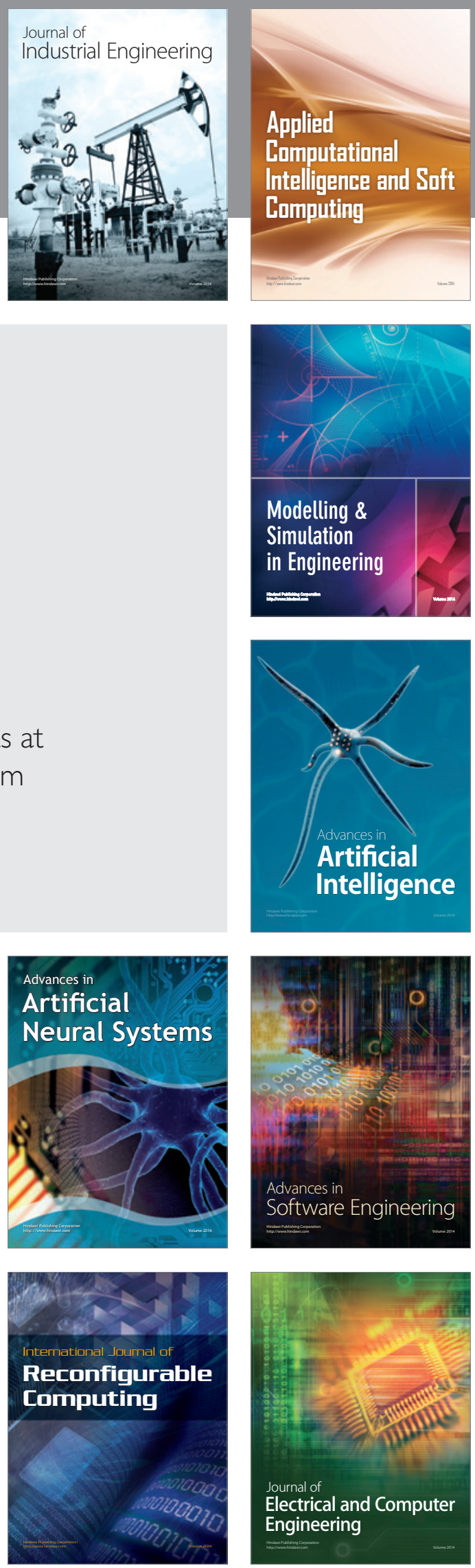